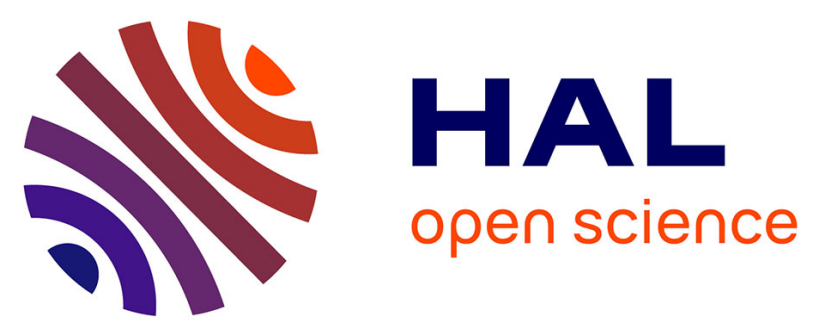

\title{
Ostéosynthèse mini-invasive de fractures extra-articulaires du radius distal : étude comparant plaque antérieure à clou centromédullaire
}

Maxence Thomas, Juan José Hidalgo Diaz, Guillaume Prunières, Sybille Facca, Yuka Igeta, Philippe Liverneaux

\section{To cite this version:}

Maxence Thomas, Juan José Hidalgo Diaz, Guillaume Prunières, Sybille Facca, Yuka Igeta, et al.. Ostéosynthèse mini-invasive de fractures extra-articulaires du radius distal: étude comparant plaque antérieure à clou centromédullaire. Revue de Chirurgie Orthopédique et Traumatologique, 2019, 105, pp.274 - 280. 10.1016/j.rcot.2018.12.003 . hal-03485021

\section{HAL Id: hal-03485021 \\ https://hal.science/hal-03485021}

Submitted on 20 Dec 2021

HAL is a multi-disciplinary open access archive for the deposit and dissemination of scientific research documents, whether they are published or not. The documents may come from teaching and research institutions in France or abroad, or from public or private research centers.
L'archive ouverte pluridisciplinaire HAL, est destinée au dépôt et à la diffusion de documents scientifiques de niveau recherche, publiés ou non, émanant des établissements d'enseignement et de recherche français ou étrangers, des laboratoires publics ou privés.

\section{(c) (1) $\$$}

Distributed under a Creative Commons Attribution - NonCommerciall 4.0 International 
Version of Record: https://www.sciencedirect.com/science/article/pii/S1877051718305495

Manuscript_5726a2d74a577a6d16593c686b4ec6fb

\section{Mémoire original}

Ostéosynthèse mini-invasive de fractures extra-articulaires du radius distal : étude comparant plaque antérieure à clou centromédullaire

Minimally invasive internal fixation for extra-articular distal radius fracture: comparison between volar plate and intramedullary nail

Maxence Thomas ${ }^{1}$, Juan José Hidalgo Diaz ${ }^{1}$, Guillaume Prunières ${ }^{1}$, Sybille Facca ${ }^{1}$, Yuka Igeta $^{1,2}$, Philippe Liverneaux $^{1}$

${ }^{1}$ Department of Hand Surgery, SOS main, CCOM, University Hospital of Strasbourg, FMTS, University of Strasbourg, Icube CNRS 7357, 10 avenue Baumann, 67400 Illkirch, France

${ }^{2}$ Department of Orthopaedic Surgery, Juntendo University Hospital, Tokyo, Japan

Auteur correspondant :

Professeur Philippe Liverneaux

Department of Hand Surgery, University Hospital of Strasbourg

10 avenue Baumann, 67403 Illkirch cedex, France

Tél 0688894779

Philippe.liverneaux@chru-strasbourg.fr

Ne pas utiliser, pour citation, la référence française de cet article, mais celle de l'article original paru dans Orthopaedics \&Traumatology: Surgery \& Research, en utilisant le DOI ci-dessus. 


\section{Résumé}

Introduction :

Les techniques d'ostéosynthèse des fractures du radius distal sont nombreuses : broches, plaques, clous. Certains ont développé des techniques mini-invasives. Le but de ce travail était de comparer 2 techniques mini-invasives d'ostéosynthèse des fractures extra-articulaires déplacées du radius distal chez des femmes âgées de plus de 50 ans par plaque antérieure ou par clou centromédullaire.

Hypothèses :

L’hypothèse principale était que la taille de l'incision après ostéosynthèse mini-invasive par plaque antérieure était inférieure à celle après ostéosynthèse mini-invasive par clou centromédullaire. Les hypothèses secondaires étaient qu'il n'y avait pas de différence entre les deux techniques en termes de douleur, scores fonctionnels, force, mobilité et index radiologiques.

Matériel-méthode :

Notre série comprenait 19 fractures A2.2 et 1 fracture A2.1 chez 20 patientes âgés en moyenne de 72 ans. Les 10 premières (groupe 1) ont été synthétisées par plaque antérieure du radius distal et les 10 dernières (groupe 2) par clou centromédullaire verrouillé sur l'épiphyse et la diaphyse du radius distal, par voie d'abord mini-invasive dans tous les cas.

Résultats :

L’hypothèse principale était vérifiée puisque la taille de l'incision après ostéosynthèse mini-invasive par plaque antérieure était inférieure à celle après par clou centromédullaire $(14,3$ contre $32,8 \mathrm{~mm}$ en moyenne). Certaines hypothèses secondaires étaient vérifiées, puisqu'il n’y avait pas de différence entre les deux techniques en termes de douleur à 6 mois, QuickDASH, PRWE, amplitudes articulaires et index radioulnaire. Deux hypothèses secondaires n'étaient pas vérifiées, puisque la douleur à 6 semaines était meilleure avec les clous et la pente palmaire meilleure avec les plaques.

Discussion et conclusion : 
L'hypothèse principale était vérifiée puisque la taille de l'incision après ostéosynthèse mini-invasive par plaque antérieure était inférieure à celle par clou centromédullaire. En conclusion, nos résultats démontrent que l'ostéosynthèse des fractures extra-articulaires instables du radius distal chez les patients de plus de 50 ans donne des résultats cliniques meilleurs à 6 semaines avec les clous centromédullaires, mais les plaques nécessitent des incisions moins grandes.

Niveau d'évidence : III, étude rétrospective

Mots clés : plaque antérieure ; fractures du radius distal ; MIPO ; mini-invasive ; clous centromédullaire 


\section{Introduction}

L'ostéosynthèse par plaque antérieure verrouillée a tendance à devenir le gold standard de l'ostéosynthèse interne des fractures instables du radius distal chez les femmes en période de périménopause [1]. Cette technique a été décrite avec une voie d’abord étendue qui nécessite une large dissection des parties molles [2]. Certains auteurs ont développé une technique mini-invasive par plaque antérieure [3] ou par clou centromédullaire [4] pour diminuer la taille des incisions. Le but de ce travail était de comparer deux techniques mini-invasives d'ostéosynthèse interne des fractures extra-articulaires instables du radius distal chez des femmes âgées de plus de 50 and par plaque antérieure ou par clou centromédullaire.

L'hypothèse principale était que la taille de l'incision après ostéosynthèse mini-invasive par plaque antérieure était inférieure à celle après ostéosynthèse mini-invasive par clou centromédullaire. Les hypothèses secondaires étaient qu'il n'y avait pas de différence entre les deux techniques en termes de douleur, scores fonctionnels, force, mobilité et index radiologiques.

\section{Matériel et méthodes}

Le Comité de Protection des Personnes local a donné son accord à la conduite de cette étude rétrospective. Les dossiers de toutes les patientes pris en charge dans notre service pour une fracture fermée extra-articulaire du radius distal à déplacement postérieur entre décembre 2016 et juin 2017 ont été étudiés rétrospectivement. Ont été exclus les patientes âgées de moins de 50 ans ou victimes de polyfractures ou polytraumatismes, les fractures articulaires, à déplacement antérieur ou ouvertes. Notre série comprenait 19 fractures A2.2 et 1 fracture A2.1 selon la classification de l'AO [5] chez 20 patientes âgées en moyenne de 72 ans avec des extrêmes de 52 et 93 (tableau I et II). 
Toutes les patientes ont été opérées sous anesthésie locorégionale en chirurgie ambulatoire et sous garrot pneumatique.

Les 10 premières fractures (groupe 1) ont été synthétisées par plaque anatomique verrouillée sur la face antérieure du radius distal (Initial $R^{\circledR}$, Newclip Technics ${ }^{T M}$, Haute-Goulaine, France) par une voie d'abord mini-invasive [3] (figure 1).

Les 10 dernières fractures (groupe 2) ont été synthétisées par clou centromédullaire verrouillé sur l'épiphyse et la diaphyse du radius distal (clou Nail-o-Flex ${ }^{\circledast}$, Evolutis $^{\top \mathrm{M}}$, Briennon, France) par une voie d'abord mini-invasive. Après incision cutanée $d^{\prime} 1 \mathrm{~cm}$ en amont de la styloïde radiale permettant de réaliser un orifice à la pointe de la stylö̈de radiale avec une mèche de $2.5 \mathrm{~mm}$, le clou monté sur un viseur était introduit à mi-distance. Après réduction de la fracture par manœuvres externes, le clou était pivoté à $180^{\circ}$ puis enfoncé jusqu'à la garde. Trois ou quatre vis transversales introduites par voie percutanée permettaient de verrouiller le clou (figure 2).

Dans les deux groupes, aucune immobilisation postopératoire n'était prescrite, et les patientes étaient encouragées à mobiliser immédiatement le membre opéré.

La durée du garrot a été mesurée en minutes.

La longueur totale de la (ou les) cicatrice(s) a été mesurée en millimètre. II n'existait qu'une seule cicatrice dans le groupe 1. II existait plusieurs cicatrices dans le groupe 2, l'une pour la mise en place du clou, et les autres pour la mise en place des vis de verrouillage.

L'évaluation a consisté à mesurer au moment de la consultation, à court terme (6 semaines) et à moyen terme (6 mois) la douleur sur une échelle numérique de 0 (pas de douleur) à 10 (douleur maximale imaginable), la fonction globale de la main par le score du QuickDASH de 0 (fonction normale) à 100 (membre supérieur inutilisable) [6], le P.R.W.E. de 0 (fonction normale) à 100 (membre supérieur inutilisable) [7], la moyenne de 3 essais de la force de la poigne en utilisant le dynamomètre de Jamar placé

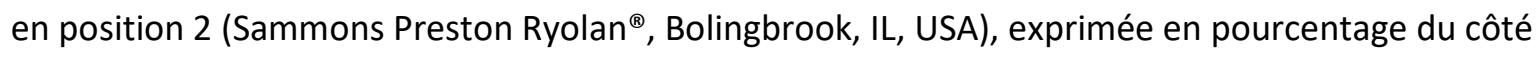
controlatéral, la mobilité passive du poignet à l'aide d'un goniomètre (flexion, extension, inclinaison ulnaire, inclinaison radiale, pronation, supination) exprimée en pourcentage du côté controlatéral, la pente 
palmaire en degrés et l'index radio-ulnaire distal en $\mathrm{mm}$. Les complications per et postopératoires ont été notées.

L'analyse statistique a consisté dans un premier temps à vérifier que les deux groupes étaient comparables en termes de dominance et d'âge du patient, du côté et du stade $\mathrm{AO}$ de la lésion. Les médianes de 16 variables quantitatives non appariées ont été comparées (durée du garrot, douleur, QuickDASH, PRWE, force de la poigne, les mobilités passives du poignet, la longueur totale de la (ou les) cicatrice(s), la pente palmaire, l'index radio-ulnaire distale). Étant donné les petits effectifs de notre échantillon, les méthodes anciennes dites « fréquentistes », exprimées sous forme de p valeurs, n'ont qu'une faible puissance. Dans ce travail ont été utilisées les méthodes d'analyses bayésiennes, qui consistent à calculer la probabilité d'observer une différence ou non, qui possèdent une plus grande puissance. Ce calcul permet d'obtenir une probabilité entre 0 et 1 , plus précise qu'une réponse binaire $p<$ ou p $>0.05$. Une probabilité de différence d'intervalles de crédibilité entre les 2 groupes de plus de $90 \%$ correspondait à forte différence, de plus de $95 \%$ à une très forte différence, et de plus de $97.5 \%$ à l'équivalent d'une différence significative. Toutes les analyses ont été réalisées à l'aide des logiciels $\mathrm{R}$ dans sa version 3.1.0 et JAGS.

\section{Résultats}

Les résultats analytiques sont présentés dans les tableaux III à VI et synthétiques dans le tableau VII.

Aucune complication peropératoire n'a été notée. En postopératoire, une rupture du long extenseur du pouce a été notée dans le groupe 1 qui a fait l'objet d'un transfert de l'extenseur propre de l'index.

La durée moyenne du garrot était de $43.3 \mathrm{~min}$ dans le groupe 1 et $54.8 \mathrm{~min}$ dans le groupe 2.

La longueur de cicatrice était de $14.3 \mathrm{~mm}$ dans le groupe 1 et $32.8 \mathrm{~mm}$ dans le groupe 2 . 
Les résultats fonctionnels étaient à 6 semaines/6 mois, respectivement pour les groupes 1 et 2 : douleur (sur 10) 3,59/0,85 et 1,74/0,76 ; QuickDASH (sur 100) 39,05/14,51 et 29,23/14,65; PRWE (sur 100) $43,14 / 17,08$ et $28,58 / 14,3$; force de poigne (en \% du côté non atteint) 52,34/78,54 et 48,22/66,06 ; flexion (en \% du côté non atteint) 77,86/91,86 et 78,09/85,76; extension 81,18/92,73 et 82,78/91,41; inclinaison ulnaire $85,93 / 92,37$ et $87,43 / 89,72$; inclinaison radiale $97,97 / 106,64$ et $90,26 / 89,73$; pronation $99,56 / 97,31$ et $107,28 / 105,66$; supination $86,46 / 92,6$ et $91,57 / 93,5$.

Les résultats radiographiques étaient à 6 semaines/6 mois, respectivement pour les groupes 1 et 2 : pente palmaire (en degrés) 10,55/10,39 et 2,14/2,69; index radio-ulnaire distal (en millimètres) -1,2/-1,1 et $0,2 / 0,03$.

\section{Discussion}

Le but de ce travail était de comparer deux techniques mini-invasives d’ostéosynthèse interne des fractures extra-articulaires instables du radius distal chez des femmes de plus de 50 ans par plaque antérieure versus par clou centromédullaire.

Dans notre étude, à court terme, les résultats cliniques étaient meilleurs pour le groupe 2 avec une différence significative concernant la douleur et une probabilité forte pour le PRWE, alors que la pente palmaire était significativement meilleure avec le groupe 1. A moyen terme, les résultats cliniques et radiologiques étaient meilleurs pour le groupe 1 avec une probabilité forte pour le Jamar et l'inclinaison radiale et une différence significative pour la pente palmaire.

L'ostéosynthèse par voie palmaire des fractures à déplacement postérieur est une méthode thérapeutique mise au point dans les années 2000 qui apporte les bénéfices de la stabilité de l'ostéosynthèse interne sans les inconvénients de l'abord dorsal [8]. Des plaques verrouillées ont été développées à cet effet pour ostéosynthéser des fractures extra-articulaires ou articulaires simples [9]. Une extension de la voie traditionnelle du flexor carpi radialis (FCR) a été décrite pour donner plus d'exposition 
au champ opératoire, réduire des déplacements articulaires importants, et libérer des adhérences à la corticale dorsale du radius de fractures vues secondairement ou de cals vicieux récents [2]. Des voies miniinvasives ont été développées pour les mêmes raisons mais dans un objectif de respect des parties molles et d'amélioration de l'aspect esthétique [10].

Toutes les études biomécaniques ont montré une supériorité des clous sur les plaques, que ce soit pour la résistance en compression ou en flexion [11, 12]. Dans la littérature on trouve des études cliniques comparant des ostéosynthèses mini-invasives par clou et des ostéosynthèses par voie conventionnelle par plaque $[13,14]$.

Concernant le temps opératoire, certains auteurs ont montré qu'il était significativement plus long pour la plaque (49 min) qu'avec le clou Targon ${ }^{\circledast}$ (45 min) [13]. Nos résultats avec le clou Nail-o-Flex ${ }^{\circledast}$ ont montré l'inverse.

Concernant les résultats cliniques, une méta-analyse ne trouve pas de différence de résultats fonctionnels au-delà de 3 mois entre les deux techniques à partir de 8 articles [14]. A court terme, certains auteurs ont montré une récupération plus rapide de l'extension et une diminution plus rapide de la douleur avec le clou Micronai ${ }^{\circledR}$ [15]. La récupération est probablement plus rapide en extension avec les clous parce que la réduction du déplacement postérieure est moins bonne qu'avec des plaques. Nos résultats cliniques n'ont pas montré de différence entre les plaques et les clous concernant l'extension du poignet.

Concernant les résultats radiologiques, certains auteurs ne retrouvent pas de différence significative avec le clou Targon ${ }^{\circledR}$ [13], contrairement à nos résultats avec le clou Nail-o-Flex ${ }^{\circledR}$ et ceux d'autres auteurs avec le clou Micronail ${ }^{\circledR}[16]$. Cela pourrait s'expliquer par l'absence d'antéversion de certains clous.

Concernant les complications, le nombre de ruptures tendineuses [16] et de canaux carpiens était plus importants avec les plaques mais les irritations de la branche sensitive du nerf radial et le nombre de vis dans I'articulation radio-ulnaire distale étaient plus importants avec le clou Targon ${ }^{\circledR}$ [13]. Dans notre série, nous avons observé une rupture du long extenseur du pouce avec une plaque et aucune complication avec les clous. 
Concernant les indications, les clous centromédullaires ont été utilisés sur des fractures extra- et intra-articulaires [14]. Notre étude portait uniquement sur des fractures extra-articulaires car le clou Nail-oFlex ${ }^{\circledR}$ ne peut accepter qu'une seule vis dans l'épiphyse et ne permet pas de fixer plusieurs fragments.

A court terme, les résultats cliniques étaient meilleurs pour le groupe 2 avec une différence significative concernant la douleur et une probabilité forte pour le PRWE, alors que la pente palmaire était significativement meilleure avec le groupe 1. Cela pourrait s'expliquer par le fait que la dissection des tissus est plus profonde avec les plaques, bien que l'incision cutanée soit plus courte qu'avec les clous. A moyen terme, les résultats cliniques et radiologiques étaient meilleurs pour le groupe 1 avec une probabilité forte pour le Jamar et l'inclinaison radiale et une différence significative pour la pente palmaire.

L'hypothèse principale était vérifiée puisque la taille de l'incision après ostéosynthèse mini-invasive par plaque antérieure était inférieure à celle par clou centromédullaire. Certaines hypothèses secondaires étaient vérifiées, puisqu'il n’y avait pas de différence entre les deux techniques en termes de douleur à 6 mois, Quick DASH, PRWE, amplitudes articulaires et index radio-ulnaire. Deux hypothèses secondaires n'étaient pas vérifiées, puisque la douleur à 6 semaines était meilleure avec les clous et la pente palmaire meilleure avec les plaques. En conclusion, nos résultats démontrent que l'ostéosynthèse des fractures extra-articulaires instables du radius distal chez les femmes de plus de 50 ans donne des résultats cliniques meilleurs à 6 semaines avec les clous centromédullaires avec moins de complications, mais les plaques nécessitent des incisions moins longues.

\section{Remerciement}

Dr Sauleau Erik, Pôle de Santé publique, Secteur méthodologie et Biostatistiques, Hôpitaux Universitaires de Strasbourg, France

\section{Conflits d'intérêts :}

Philippe Liverneaux a des conflits d'intérêt avec Newclip Technics, Argomedical, Zimmer Biomet, Biomodex Aucun des autres auteurs n'a de conflit à déclarer 
Sources de financement : aucune

\section{Contribution des auteurs :}

Maxence Thomas : a revu les dossiers

Juan José Hidalgo Diaz, Guillaume Prunières, Sybille Facca ont opéré les patients

Yuka Igeta a revu le manuscrit

Philippe Liverneaux a participé à toutes les étapes 


\section{References}

1. Gehrmann SV, Windolf J, Kaufmann RA. Distal radius fracture management in elderly patients: a literature review. J Hand Surg Am. 2008;33:421-529.

2. Wijffels MM, Orbay JL, Indriago I, Ring D. The extended flexor carpi radialis approach for partially healed malaligned fractures of the distal radius. Injury. 2012;43:1204-1208.

3. Lebailly F, Zemirline A, Facca S, Gouzou S, Liverneaux P. Distal radius fixation through a miniinvasive approach of $15 \mathrm{~mm}$. Part 1: a series of 144 cases. Eur J Orthop Surg Traumatol. 2014;24:877-890.

4. Chappuis J, Bouté P, Putz P. Dorsally displaced extra-articular distal radius fractures fixation: Dorsal IM nailing versus volar plating. A randomized controlled trial. Orthop Traumatol Surg Res. 2011;97:471-478. 5. Müller ME, Nazarian S, Koch P. Classification AO des fractures. Tome I les os longs. 1st ed Berlin Springer-Verlag. 1987

6. Gummesson C, Ward MM, Atroshi I. The shortened disabilities of the arm, shoulder and hand questionnaire (QuickDASH): validity and reliability based on responses within the full length DASH. BMC Musculoskelet Disorder, 2006;7:44.

7. MacDermid JC, Turgeon T, Richards RS et al. Patient rating of wrist pain and disability: a reliable and valid measurement tool. J Orthop Trauma 1998;12:577-586.

8. Orbay JL. The treatment of unstable distal radius fractures with volar fixation. Hand Surg. 2000; 5: 103-12.

9. Orbay JL, Fernandez DL. Volar fixation for dorsally displaced fractures of the distal radius: a preliminary report. J Hand Surg Am. 2002; 27: 205-15.

10. Liverneaux P, Ichihara S, Facca S, Hidalgo Diaz JJ. Outcomes of minimally invasive plate osteosynthesis (MIPO) with volar locking plates in distal radius fractures: A review. Hand Surg Rehabil. 2016; 35S: S80-S85.

11. Burkhart KJ, Nowak TE, Gradl G, Klitscher D, Mehling I, Mehler D, Mueller LP, Rommens PM. Intramedullary nailing vs. palmar locked plating for unstable dorsally comminuted distal radius fractures: a biomechanical study. Clin Biomech (Bristol, Avon). 2010;25:771-775. 
12. Konstantinidis L, Helwig P, Seifert J, Hirschmüller A, Liodakis E, Südkamp NP, Oberst M. Internal fixation of dorsally comminuted fractures of the distal part of the radius: a biomechanical analysis of volar plate and intramedullary nail fracture stability. Arch Orthop Trauma Surg. 2011;131:1529-1537.

13. Gradl G, Mielsch N, Wendt M, Falk S, Mittlmeier T, Gierer P, Gradl G. Intramedullary nail versus volar plate fixation of extra-articular distal radius fractures. Two year results of a prospective randomized trial. Injury. 2014;45:S3-S8.

14. Zhang B, Chang H, Yu K, Bai J, Tian D, Zhang G, Shao X, Zhang Y. Intramedullary nail versus volar locking plate fixation for the treatment of extra-articular or simple intra-articular distal radius fractures: systematic review and meta-analysis. Int Orthop. 2017:41: 2161-2169.

15. Plate JF, Gaffney DL, Emory CL, Mannava S, Smith BP, Koman LA, Wiesler ER, Li Z. Randomized comparison of volar locking plates and intramedullary nails for unstable distal radius fractures. J Hand Surg Am. 2015;40:1095-1101.

16. Nishiwaki M1, Tazaki K, Shimizu H, Ilyas AM. Prospective study of distal radial fractures treated with an intramedullary nail. J Bone Joint Surg Am. 2011;93:1436-1441. 


\section{Légendes des Figures}

Figure 1 Technique de fixation d'une fracture du radius distal AO2.2 par plaque antérieure verrouillée (Initial $R^{\circledast}$, Newclip Technics ${ }^{\top M}$, Haute-Goulaine, France) (patient $n^{\circ}$ )
A radiographie préopératoire
B radiographie postopératoire
C incision au dernier recul mesurée à $14 \mathrm{~mm}$

Figure 2 Technique de fixation d'une fracture du radius distal AO2.2 par clou centromédullaire verrouillé (clou Nail-o-Flex ${ }^{\circledast}$, Evolutis $^{\top \mathrm{M}}$, Briennon, France) (patient $\mathrm{n}^{\circ} 12$ )
A radiographie préopératoire
B radiographie postopératoire
C incision au dernier recul mesurée à $30 \mathrm{~mm}$ avec la rétraction cutanée 


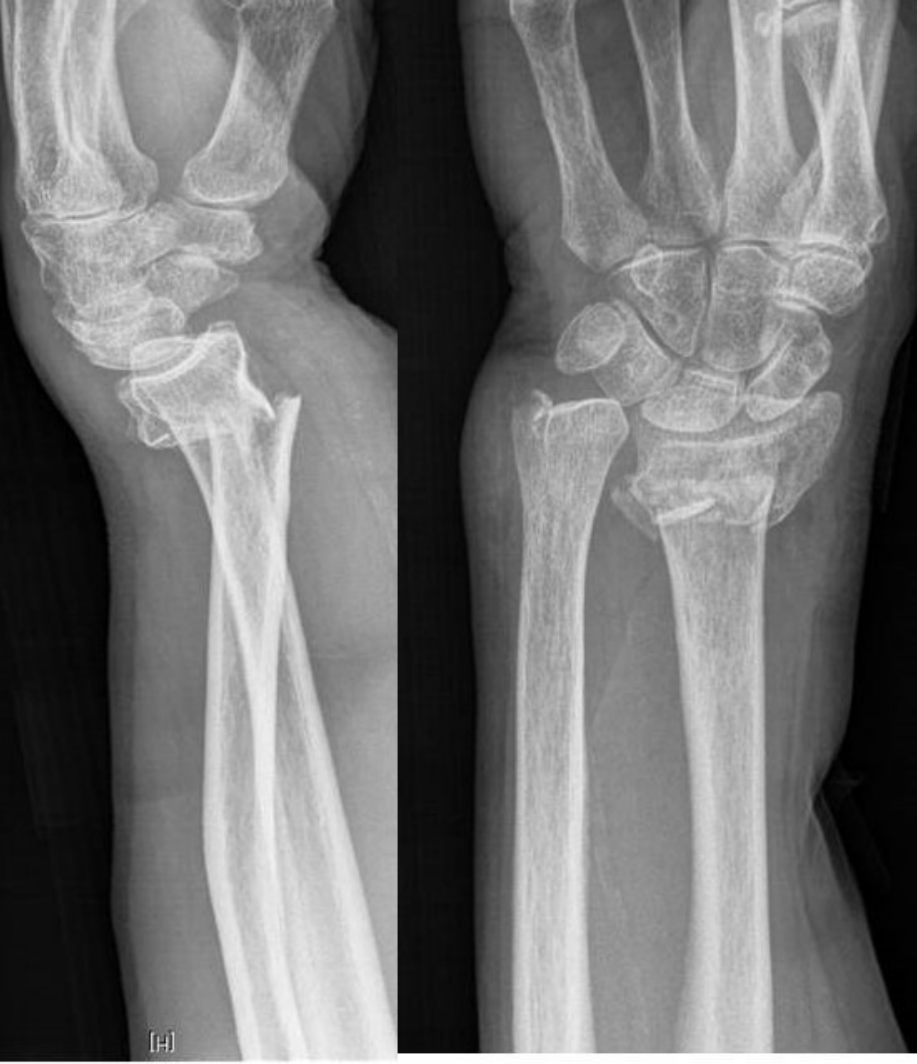




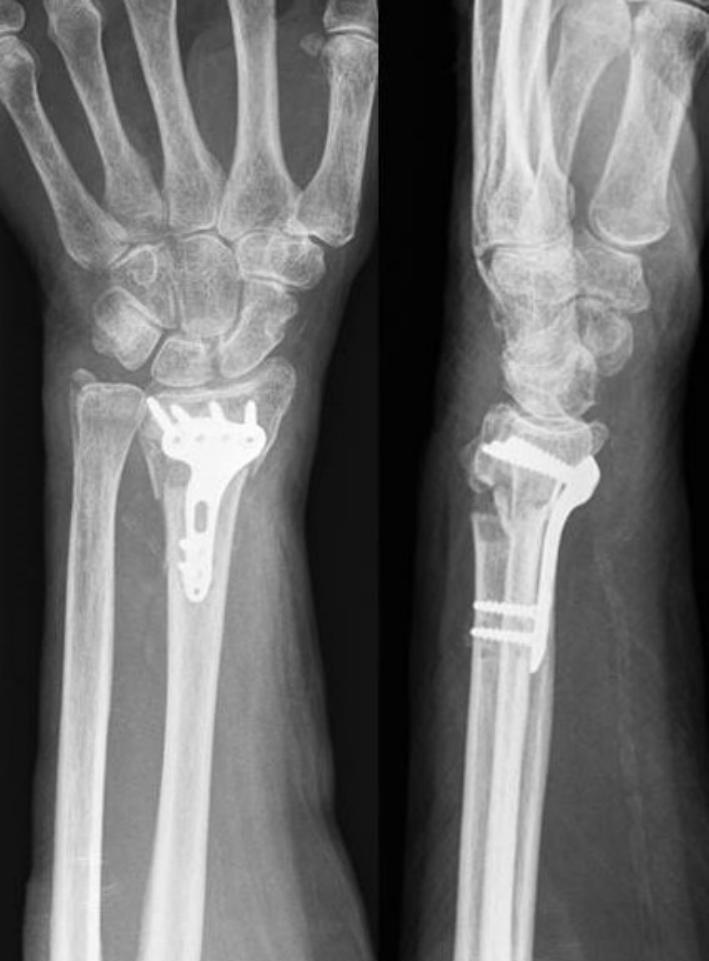




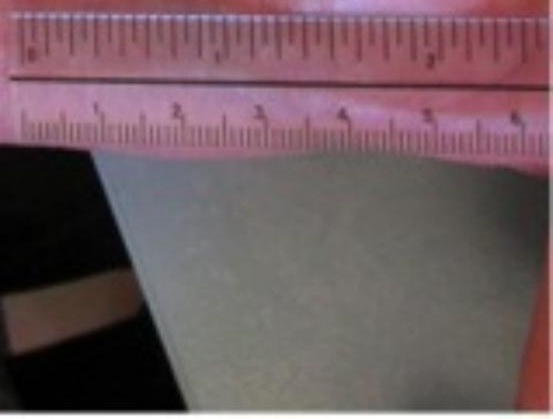




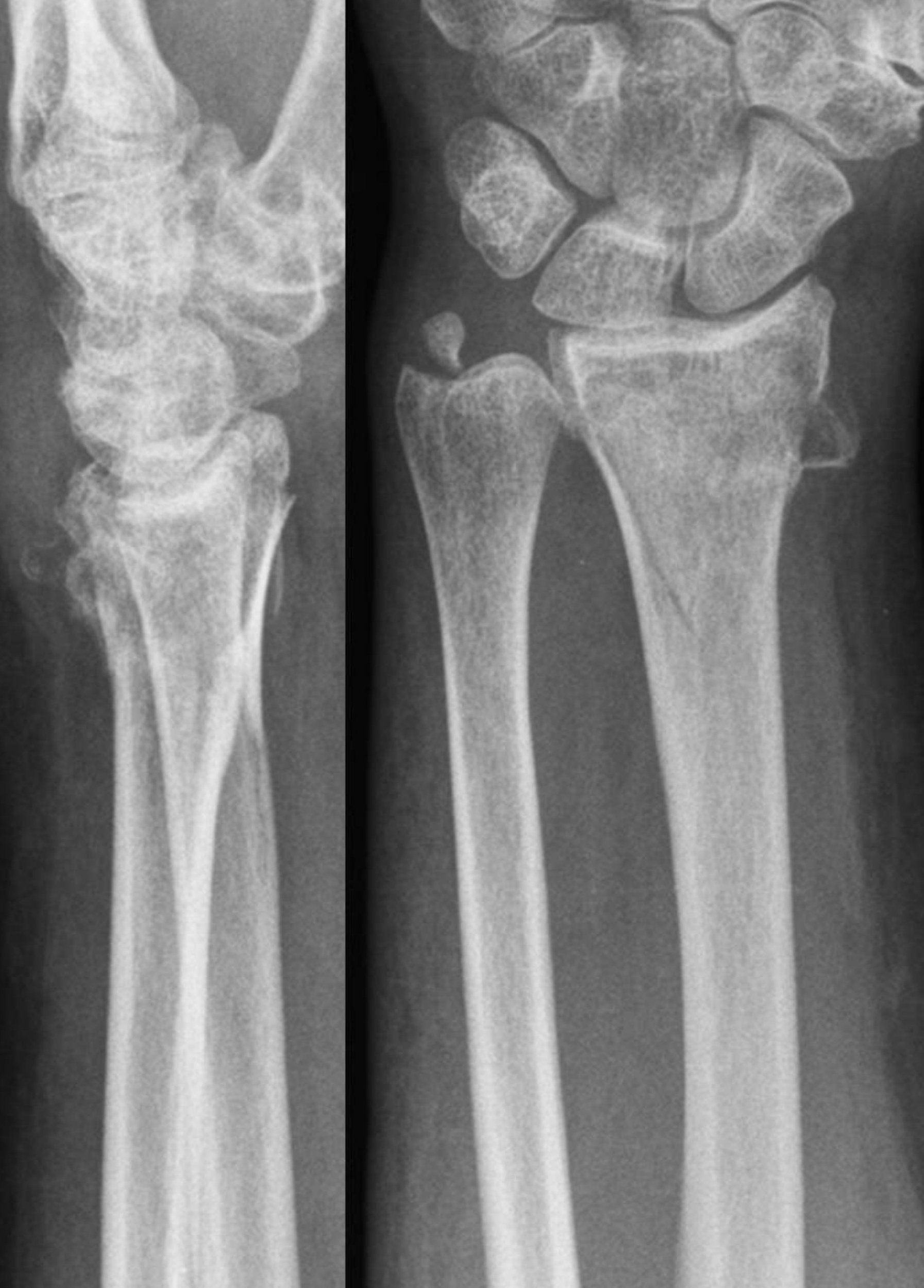



Tableau I. Casuistique d'une série de 10 fractures extra-articulaires du radius distal opérées par plaque Initial $\mathbf{R}^{\circledR}$

\begin{tabular}{|c|c|c|c|c|c|}
\hline $\begin{array}{c}\text { Patient } \\
(\mathrm{n})\end{array}$ & $\begin{array}{l}\text { Age } \\
\text { (ans) }\end{array}$ & $\begin{array}{c}\text { Latéralité } \\
(D / G)\end{array}$ & $\begin{array}{l}\text { Côté atteint } \\
\qquad(D / G)\end{array}$ & $\begin{array}{l}\text { Classification } A O \\
\text { (A2.1/A2.2/A2.3) }\end{array}$ & $\begin{array}{c}\text { Durée du garrot } \\
\text { (min) }\end{array}$ \\
\hline 1 & 82 & $D$ & D & $\mathrm{A} 2.2$ & 44 \\
\hline 2 & 86 & G & G & A 2.2 & 33 \\
\hline 3 & 71 & D & G & A 2.2 & 24 \\
\hline 4 & 79 & G & G & A 2.2 & 39 \\
\hline 5 & 70 & $D$ & G & A 2.2 & 57 \\
\hline 6 & 68 & $D$ & G & A 2.2 & 40 \\
\hline 7 & 85 & $D$ & G & A 2.2 & 84 \\
\hline 8 & 78 & D & G & A 2.2 & 30 \\
\hline 9 & 52 & $D$ & D & A 2.2 & 51 \\
\hline 10 & 61 & $D$ & D & A 2.2 & 31 \\
\hline
\end{tabular}

D droit. G gauche 
Tableau II. Casuistique d’une série de 10 fractures extra-articulaires du radius distal opérées par clou Nail-o-Flex ${ }^{\circledR}$

\begin{tabular}{c|c|c|c|c|c}
$\begin{array}{c}\text { Patient } \\
(\mathrm{n})\end{array}$ & $\begin{array}{c}\text { Age } \\
(\mathrm{ans})\end{array}$ & $\begin{array}{c}\text { Latéralité } \\
(\mathrm{D} / \mathrm{G})\end{array}$ & $\begin{array}{c}\text { Côté atteint } \\
(\mathrm{D} / \mathrm{G})\end{array}$ & $\begin{array}{c}\text { Classification AO } \\
(\mathrm{A} 2.1 / \mathrm{A} 2.2 / \mathrm{A} 2.3)\end{array}$ & $\begin{array}{c}\text { Durée du garrot } \\
(\mathrm{min})\end{array}$ \\
\hline 11 & 76 & $\mathrm{D}$ & $\mathrm{D}$ & $\mathrm{A} 2.2$ & 47 \\
12 & 59 & $\mathrm{D}$ & $\mathrm{G}$ & $\mathrm{A} 2.2$ & 65 \\
13 & 84 & $\mathrm{D}$ & $\mathrm{G}$ & $\mathrm{A} 2.1$ & 60 \\
14 & 69 & $\mathrm{D}$ & $\mathrm{D}$ & $\mathrm{A} 2.2$ & 44 \\
15 & 69 & $\mathrm{D}$ & $\mathrm{D}$ & $\mathrm{A} 2.2$ & 70 \\
16 & 60 & $\mathrm{D}$ & $\mathrm{G}$ & $\mathrm{A} 2.2$ & 58 \\
17 & 66 & $\mathrm{D}$ & $\mathrm{G}$ & $\mathrm{A} 2.2$ & 30 \\
18 & 53 & $\mathrm{D}$ & $\mathrm{G}$ & $\mathrm{A} 2.2$ & 65 \\
19 & 71 & $\mathrm{D}$ & $\mathrm{D}$ & $\mathrm{A} 2.2$ & 50 \\
20 & 93 & $\mathrm{D}$ & $\mathrm{D}$ & $\mathrm{A} 2.2$ & 59
\end{tabular}

D droit. G gauche 
Tableau III. Résultats à court terme d’une série de 10 fractures extra-articulaires du radius distal opérées par plaque Initial $\mathbf{R}^{\circledR}$

\begin{tabular}{|c|c|c|c|c|c|c|c|c|c|c|c|c|c|c|c|}
\hline \multirow[t]{2}{*}{$\begin{array}{l}\text { Patient } \\
\text { (n) }\end{array}$} & \multirow[t]{2}{*}{$\begin{array}{l}\text { Recul } \\
\text { (mois) }\end{array}$} & \multirow[t]{2}{*}{$\begin{array}{l}\text { Douleur } \\
(0-10)\end{array}$} & \multirow[t]{2}{*}{$\begin{array}{l}\text { QuickDASH } \\
(0-100)\end{array}$} & \multirow[t]{2}{*}{$\begin{array}{l}\text { PRWE } \\
(0-100)\end{array}$} & \multirow[t]{2}{*}{$\begin{array}{c}\text { Jamar } \\
\%\end{array}$} & \multicolumn{6}{|c|}{$\begin{array}{c}\text { Mobilité passive } \\
\%\end{array}$} & \multirow[t]{2}{*}{$\begin{array}{l}\text { Taille incision } \\
\qquad(\mathrm{mm})\end{array}$} & \multirow[t]{2}{*}{$\begin{array}{l}\text { Pente palmaire } \\
\left({ }^{\circ}\right)\end{array}$} & \multirow[t]{2}{*}{$\begin{array}{l}\text { Index RUD } \\
\qquad(\mathrm{mm})\end{array}$} & \multirow[t]{2}{*}{ complications } \\
\hline & & & & & & $\mathrm{F}$ & $E$ & UI & $\mathrm{RI}$ & $P$ & S & & & & \\
\hline 1 & 1.5 & 4 & 59.09 & 54 & 50 & 75 & 57 & 71 & 67 & 100 & 100 & 15 & 13 & 0 & 0 \\
\hline 2 & 1.5 & 3 & 22.73 & 29 & 82 & 100 & 100 & 87 & 100 & 100 & 100 & 12 & 3 & $-3,5$ & 0 \\
\hline 3 & 1.5 & 7 & 77.27 & 54 & 25 & 70 & 56 & 86 & 50 & 70 & 47 & 14 & 13 & 0 & 0 \\
\hline 4 & 1.5 & 7 & 59.09 & 95 & 27 & 71 & 93 & 100 & 100 & 100 & 100 & 15 & 2 & 0 & 0 \\
\hline 5 & 1.5 & 2 & 31.82 & 39 & 85 & 62 & 70 & 100 & 100 & 94 & 94 & 16 & 8 & -1 & adhérence LFP \\
\hline 6 & 1.5 & 2 & 52.27 & 30 & 57 & 94 & 75 & 100 & 100 & 100 & 100 & 10 & 10 & -2.5 & 0 \\
\hline 7 & 1.5 & 1 & 13.64 & 11 & 36 & 75 & 75 & 75 & 80 & 94 & 71 & 14 & 3 & 0 & 0 \\
\hline 8 & 1.5 & 3 & 15.91 & 27 & 47 & 41 & 63 & 57 & 100 & 100 & 35 & 14 & 10 & 0 & 0 \\
\hline 9 & 1.5 & 6 & 45.45 & 17 & 55 & 75 & 100 & 75 & 200 & 100 & 75 & 17 & 12 & +2 & 0 \\
\hline 10 & 1.5 & 3 & 61.36 & 85 & 36 & 78 & 85 & 75 & 75 & 100 & 100 & 12 & 14 & -2 & 0 \\
\hline
\end{tabular}

$\%=\%$ par rapport au côté controlatéral sain. $\mathrm{RUD}=$ radio-ulnaire distal. $\mathrm{F}=$ flexion. $\mathrm{E}=$ extension. $\mathrm{UI}=$ inclinaison ulnaire. $\mathrm{RI}=$ inclinaison radiale. $\mathrm{P}=$

Pronation. $\mathrm{S}=$ Supination.. LFP = long fléchisseur du pouce 
Tableau IV. Résultats à court terme d'une série de 10 fractures extra-articulaires du radius distal opérées par clou Nail-o-Flex ${ }^{\circledR}$

\begin{tabular}{|c|c|c|c|c|c|c|c|c|c|c|c|c|c|c|c|c|c|c|c|c|}
\hline \multirow[t]{2}{*}{$\begin{array}{l}\text { Patient } \\
\text { (n) }\end{array}$} & \multirow[t]{2}{*}{$\begin{array}{l}\text { Recul } \\
\text { (mois) }\end{array}$} & \multirow[t]{2}{*}{$\begin{array}{l}\text { Douleur } \\
(0-10)\end{array}$} & \multirow[t]{2}{*}{$\begin{array}{l}\text { QuickDASH } \\
(0-100)\end{array}$} & \multirow{2}{*}{$\begin{array}{l}\text { PRWE } \\
(0- \\
100)\end{array}$} & \multirow[t]{2}{*}{$\begin{array}{l}\text { Jamar } \\
\%\end{array}$} & \multicolumn{6}{|c|}{ Mobilité passive } & \multicolumn{6}{|c|}{ Taille incision } & \multirow[t]{2}{*}{$\begin{array}{c}\text { Pente } \\
\text { palmaire } \\
\left(^{\circ}\right)\end{array}$} & \multirow[t]{2}{*}{$\begin{array}{l}\text { Index } \\
\text { RUD } \\
(\mathrm{mm})\end{array}$} & \multirow[t]{2}{*}{ complications } \\
\hline & & & & & & $\mathrm{F}$ & $E$ & UI & $\mathrm{RI}$ & $P$ & $S$ & $A$ & B & C & D & $E$ & total & & & \\
\hline 11 & 2 & 6 & 84.09 & 79 & 30 & 64 & 37 & 75 & 75 & 83 & 94 & 15 & 5 & 0 & 0 & 20 & 40 & +4 & 0 & 0 \\
\hline 12 & 1,5 & 2 & 50 & 58 & 12,5 & 33 & 60 & 50 & 100 & 94 & 61 & 5 & 5 & 0 & 0 & 27 & 37 & 1 & -2.5 & 0 \\
\hline 13 & 1,5 & 1 & 15.91 & 17 & 57 & 54 & 122 & 86 & 50 & 100 & 67 & 4 & 6 & 0 & 0 & 21 & 31 & -2 & +2.5 & 0 \\
\hline 14 & 1.5 & 0 & 13.64 & 11 & 100 & 110 & 77 & 100 & 50 & 160 & 77 & 3 & 3 & 0 & 0 & 25 & 31 & +7 & +1 & 0 \\
\hline 15 & 1.5 & 1 & 15.91 & 17 & 50 & 89 & 77 & 100 & 100 & 87 & 83 & 10 & 16 & 5 & 5 & 5 & 41 & +10 & +2.5 & 0 \\
\hline 16 & 1.5 & 0 & 18.18 & 6 & 31 & 90 & 84 & 100 & 100 & 100 & 100 & 8 & 8 & 0 & 0 & 10 & 26 & +5 & 0 & 0 \\
\hline 17 & 1.5 & 2 & 40.91 & 31 & 13 & 37 & 53 & 57 & 50 & 93 & 61 & 12 & 10 & 0 & 0 & 12 & 32 & -10 & +2 & 0 \\
\hline 18 & 1.5 & 2 & 40.91 & 27 & 50 & 36 & 53 & 50 & 75 & 93 & 75 & 5 & 5 & 20 & 0 & 5 & 35 & +4 & -1.8 & 0 \\
\hline 19 & 1.5 & 2 & 40.91 & 31 & 30 & 44 & 19 & 57 & 50 & 100 & 100 & 5 & 10 & 0 & 0 & 10 & 25 & -4 & +3 & 0 \\
\hline 20 & 1.5 & 2 & 18.18 & 11 & 30 & 78 & 82 & 86 & 50 & 100 & 100 & 5 & 5 & 5 & 5 & 10 & 30 & -5 & +2 & 0 \\
\hline
\end{tabular}

$\%=\%$ par rapport au côté controlatéral sain. $\mathrm{RUD}=$ radio-ulnaire distal. $\mathrm{F}=$ flexion. $\mathrm{E}=$ extension. $\mathrm{UI}=$ inclinaison $u l n a i r e . \mathrm{RI}=$ inclinaison radiale. $\mathrm{P}=$ Pronation. S = Supination.. 
Tableau V. Résultats à moyen terme d'une série de 10 fractures extra-articulaires du radius distal opérées par plaque Initial $\mathbf{R}^{\circledR}$

\begin{tabular}{|c|c|c|c|c|c|c|c|c|c|c|c|c|c|c|c|}
\hline \multirow{3}{*}{$\begin{array}{l}\text { Patient } \\
\text { (n) }\end{array}$} & \multirow{3}{*}{$\begin{array}{l}\text { Recul } \\
\text { (mois) }\end{array}$} & \multirow{3}{*}{$\begin{array}{l}\text { douleur } \\
(0-10)\end{array}$} & \multirow{3}{*}{$\begin{array}{c}\text { QuickDASH } \\
(0-100)\end{array}$} & \multirow{3}{*}{$\begin{array}{l}\text { PRWE } \\
(0-100)\end{array}$} & \multirow{3}{*}{$\begin{array}{l}\text { Jamar } \\
\text { \% }\end{array}$} & \multicolumn{6}{|c|}{ Mobilité passive } & \multirow{3}{*}{$\begin{array}{l}\text { Taille incision } \\
(\mathrm{mm})\end{array}$} & \multirow{3}{*}{$\begin{array}{l}\text { Pente palmaire } \\
\qquad\left(^{\circ}\right)\end{array}$} & \multirow{3}{*}{$\begin{array}{l}\text { Index RUD } \\
\text { (mm) }\end{array}$} & \multirow{3}{*}{ complications } \\
\hline & & & & & & & & & & & & & & & \\
\hline & & & & & & $\mathrm{F}$ & $\mathrm{E}$ & UI & $\mathrm{RI}$ & $\mathrm{P}$ & $S$ & & & & \\
\hline 1 & 7 & 1 & 27.27 & 15 & 88 & 92 & 80 & 75 & 100 & 87 & 100 & 15 & 13 & 0 & 0 \\
\hline 2 & 7 & 2 & 16.64 & 13 & 85 & 100 & 100 & 100 & 100 & 100 & 100 & 12 & 3 & -3.5 & 0 \\
\hline 3 & 6 & 0 & 20.45 & 20 & 40 & 82 & 75 & 86 & 75 & 94 & 82 & 14 & 13 & +1 & 0 \\
\hline 4 & 7 & 2 & 20.45 & 20 & 67 & 87 & 93 & 100 & 100 & 100 & 100 & 15 & 2 & 0 & 0 \\
\hline 5 & 6 & 0 & 18.18 & 19 & 119 & 93 & 77 & 100 & 100 & 94 & 94 & 16 & 9 & -1 & Rupture LEP \\
\hline 6 & 6 & 0 & 2.27 & 5 & 122 & 100 & 88 & 100 & 100 & 100 & 100 & 10 & 6 & -3 & 0 \\
\hline 7 & 6 & 0 & 0 & 4 & 55 & 88 & 100 & 100 & 100 & 67 & 67 & 14 & 3 & 0 & 0 \\
\hline 8 & 6 & 0 & 6.82 & 5 & 69 & 94 & 100 & 85 & 100 & 100 & 82 & 14 & 10 & 0 & 0 \\
\hline 9 & 6 & 1 & 20.45 & 13 & 55 & 75 & 100 & 75 & 200 & 107 & 88 & 15 & 13 & +2.5 & 0 \\
\hline 10 & 6 & 1 & 15.91 & 32 & 112 & 100 & 100 & 88 & 100 & 100 & 100 & 12 & 14 & -2 & 0 \\
\hline
\end{tabular}

$\%=\%$ par rapport au côté controlatéral sain. $\mathrm{RUD}=$ radio-ulnaire distal. $\mathrm{F}=$ flexion. $\mathrm{E}=$ extension. $\mathrm{UI}=$ inclinaison ulnaire. $\mathrm{RI}$ inclinaison radiale. $\mathrm{P}=$ Pronation. $\mathrm{S}=$ Supination.. LEP = long extenseur du pouce 
Tableau VI. Résultats à moyen terme d'une série de 10 fractures extra-articulaires du radius distal opérées par clou Nail-o-Flex ${ }^{\circledR}$

\begin{tabular}{|c|c|c|c|c|c|c|c|c|c|c|c|c|c|c|c|c|c|c|c|c|}
\hline \multirow[t]{2}{*}{$\begin{array}{l}\text { Patient } \\
\text { (n) }\end{array}$} & \multirow[t]{2}{*}{$\begin{array}{l}\text { Recul } \\
\text { (mois) }\end{array}$} & \multirow[t]{2}{*}{$\begin{array}{l}\text { Douleur } \\
\text { (0-10) }\end{array}$} & \multirow[t]{2}{*}{$\begin{array}{c}\text { QuickDASH } \\
(0-100)\end{array}$} & \multirow{2}{*}{$\begin{array}{l}\text { PRWE } \\
(0- \\
100)\end{array}$} & \multirow[t]{2}{*}{$\begin{array}{l}\text { Jamar } \\
\qquad \%\end{array}$} & \multicolumn{6}{|c|}{ Mobilité passive } & \multicolumn{6}{|c|}{$\begin{array}{l}\text { Taille incision } \\
\text { (mm) }\end{array}$} & \multirow[t]{2}{*}{$\begin{array}{c}\text { Pente } \\
\text { palmaire } \\
\left({ }^{\circ}\right)\end{array}$} & \multirow[t]{2}{*}{$\begin{array}{l}\text { Index } \\
\text { RUD } \\
(\mathrm{mm})\end{array}$} & \multirow[t]{2}{*}{ complications } \\
\hline & & & & & & $\mathrm{F}$ & $\mathrm{E}$ & UI & $\mathrm{RI}$ & $P$ & $S$ & A & B & C & $D$ & $E$ & total & & & \\
\hline 11 & 6 & 2 & 50 & 16 & 50 & 92 & 75 & 87 & 50 & 92 & 75 & 15 & 5 & 0 & 0 & 20 & 40 & $+6^{\circ}$ & +1 & 0 \\
\hline 12 & 6 & 1 & 34.09 & 33 & 60 & 40 & 73 & 63 & 50 & 95 & 78 & 5 & 5 & 0 & 0 & 27 & 37 & 1.5 & -2 & 0 \\
\hline 13 & 6 & 0 & 6.82 & 10 & 62 & 72 & 122 & 100 & 50 & 100 & 83 & 4 & 6 & 0 & 0 & 21 & 31 & 0 & +2.5 & 0 \\
\hline 14 & 6 & 0 & 0 & 0 & 78 & 100 & 100 & 50 & 100 & 100 & 75 & 3 & 3 & 0 & 0 & 25 & 31 & +8 & +1 & 0 \\
\hline 15 & 6 & 0 & 13.64 & 25 & 75 & 100 & 67 & 100 & 100 & 106 & 89 & 10 & 16 & 5 & 5 & 5 & 41 & +6 & +1.5 & 0 \\
\hline 16 & 6 & 0 & 0 & 0 & 67 & 79 & 100 & 100 & 50 & 86 & 100 & 8 & 8 & 0 & 0 & 10 & 26 & +3 & -1 & 0 \\
\hline 17 & 6 & 1 & 6.82 & 10 & 50 & 52 & 60 & 71 & 75 & 100 & 78 & 12 & 10 & 0 & 0 & 12 & 32 & -10 & +2 & 0 \\
\hline 18 & 6 & 1 & 25 & 9 & 90 & 64 & 93 & 60 & 100 & 106 & 78 & 5 & 5 & 20 & 0 & 5 & 35 & +8 & -2 & 0 \\
\hline 19 & 6 & 1 & 34.09 & 21 & 57 & 83 & 56 & 67 & 60 & 87 & 100 & 5 & 10 & 0 & 0 & 10 & 25 & +2.5 & +2 & 0 \\
\hline 20 & 6 & 0 & 9.09 & 9 & 86 & 82 & 91 & 100 & 100 & 87 & 78 & 5 & 5 & 5 & 5 & 10 & 30 & -9 & 2 & 0 \\
\hline
\end{tabular}

$\%=\%$ par rapport au côté controlatéral sain. $\mathrm{RUD}=$ radio-ulnaire distal. $\mathrm{F}=$ flexion. $\mathrm{E}=$ extension. $\mathrm{UI}=$ inclinaison ulnaire. $\mathrm{RI}=$ inclinaison radiale. $\mathrm{P}=$ Pronation. S = Supination.. 
Tableau VII. Résultats à court et à moyen terme d'une série de 20 fractures extra-articulaires du radius distal opérées par plaque Initial $R^{\circledR}$ (groupe 1 ) et clou Nail-o-Flex ${ }^{\circledR}$ (groupe 2)

\begin{tabular}{|c|c|c|c|c|c|c|c|c|c|c|c|c|c|c|}
\hline \multirow[t]{3}{*}{ groupe } & \multicolumn{14}{|c|}{ Mediane à court terme } \\
\hline & \multirow{2}{*}{$\begin{array}{l}\text { Durée du } \\
\text { garrot } \\
\text { (min) }\end{array}$} & \multirow{2}{*}{$\begin{array}{c}\text { Douleur } \\
(0-10)\end{array}$} & \multirow{2}{*}{$\begin{array}{c}\text { QuickDASH } \\
(0-100)\end{array}$} & \multirow{2}{*}{$\begin{array}{l}\text { PRWE } \\
(0-100)\end{array}$} & \multirow{2}{*}{$\begin{array}{c}\text { Jamar } \\
\%\end{array}$} & \multicolumn{6}{|c|}{ Mobilité passive } & Taille incision & \multirow{2}{*}{$\begin{array}{l}\text { Pente } \\
\text { palmaire } \\
\left(^{\circ}\right)\end{array}$} & \multirow{2}{*}{$\begin{array}{l}\text { Index RUD } \\
\text { (mm) }\end{array}$} \\
\hline & & & & & & $F$ & $E$ & UI & $\mathrm{RI}$ & $P$ & $s$ & $\begin{array}{l}(\mathrm{mm}) \\
\text { Total }\end{array}$ & & \\
\hline 1 & 45,320 & 3.591 & $39.050[8.496 ;$ & $43.142[8.416 ;$ & 52.339 & 77.860 & 81.185 & 85.933 & 97.967 & 99.560 & 86.457 & 13,461 & +10.546 & \\
\hline & $\begin{array}{l}{[29,325 ;} \\
61,431]\end{array}$ & $\begin{array}{l}{[0.663 ;} \\
7.942]\end{array}$ & $80.637]$ & 83.923] & $\begin{array}{l}{[34.346 ;} \\
70.078]\end{array}$ & $\begin{array}{l}\text { [67.115; } \\
89.053]\end{array}$ & $\begin{array}{l}{[69.486 ;} \\
93.341]\end{array}$ & $\begin{array}{l}\text { [75.706; } \\
96.754]\end{array}$ & $\begin{array}{l}\text { [82.991; } \\
\text { 113.159] }\end{array}$ & [89.187; & [75.715; & {$[4,770$} & {$[1.142 ;$} & $\begin{array}{l}2.640 ; \\
0.271\end{array}$ \\
\hline 2 & 54,168 & 1.744 & 29.235 [6.614; & 28.582 & 48.221 & $\begin{array}{l}89.05331 \\
78.092\end{array}$ & $\begin{array}{l}93.341 \mathrm{~J} \\
82.777\end{array}$ & $\begin{array}{l}96.6754] \\
87.433\end{array}$ & $\begin{array}{c}113.1 .259] \\
90.260\end{array}$ & $\begin{array}{l}110.587] \\
107.280\end{array}$ & $\begin{array}{l}{ }_{97.587]} \\
91.573\end{array}$ & $\begin{array}{l}22,245] \\
31,760\end{array}$ & $\begin{array}{c}19.950] \\
2.142[-6.099 ;\end{array}$ & $\begin{array}{r}0.271] \\
+0.198[-\end{array}$ \\
\hline & $\begin{array}{l}{[40,741 ;} \\
67,404]\end{array}$ & $\begin{array}{l}{[0.260 ;} \\
5.223]\end{array}$ & $66.993]$ & {$[5.157 ; 65.672]$} & $\begin{array}{l}{[33.942 ;} \\
62.248]\end{array}$ & $\begin{array}{l}{[68.474 ;} \\
88.445]\end{array}$ & $\begin{array}{l}\text { [72.618; } \\
93.478]\end{array}$ & $\begin{array}{l}{[77.861 ;} \\
97.211]\end{array}$ & $\begin{array}{l}{[78.020 ;} \\
102.698]\end{array}$ & $\begin{array}{l}\text { [97.675; } \\
\text { 117.132] }\end{array}$ & $\begin{array}{l}\text { [82.028; } \\
101.657]\end{array}$ & $\begin{array}{l}{[23,645 ;} \\
39,761]\end{array}$ & $10.404]$ & $\begin{array}{l}1.440 \\
1.843\end{array}$ \\
\hline différence & 1,714 & 1.846 & 9.815 & 15.142 & 4,118 & -0.232 & -1.593 & -1.5 & 7.707 & -7.719 & -5.116 & -18.324 & 8.404 & -1.397 \\
\hline Intervalle de & {$[-2.593 ;$} & {$[0.177 ;$} & {$[-7.602 ;$} & {$[-3.368 ; 37.224]$} & {$[-9,455 ;$} & {$[-10.950 ;$} & {$[-13.302 ;$} & {$[-12.004 ;$} & {$[-6.658 ;$} & {$[-18.271 ;$} & {$[-16.050 ;$} & {$[-22.538 ;]$} & {$[3.645 ;$} & {$[-3.148$} \\
\hline confiance & 25.593] & 4.112] & 30.101] & & $17,363]$ & 10.335] & 9.819] & 8.951] & 22.041] & 3.060] & $5.363]$ & 13.593] & 13.067] & $0.372]$ \\
\hline \multirow{2}{*}{$\begin{array}{l}\text { Probabilitié de } \\
\text { différence }\end{array}$} & $89.6 \%$ & $99,1 \%$ & $86,1 \%$ & $94,2 \%$ & $73,4 \%$ & $48,7 \%$ & $40,0 \%$ & $38,6 \%$ & $85,5 \%$ & $7.6 \%$ & $17.4 \%$ & $99.9 \%$ & 99.9\% & $5.7 \%$ \\
\hline & \multicolumn{14}{|c|}{ Mediane à moyen terme } \\
\hline 1 & & & & $17.084[1.791 ;$ & & & 92.730 & 92.373 & 103.636 & 97.315 & 92.604 & & $\begin{array}{ll}10.388 \\
\end{array}$ & \\
\hline & & {$[0.084$} & 47.100] & $53.061]$ & [58.664; & {$[80.329 ;$} & {$[79.736$} & [81.192; & {$[85.520$} & [86.063; & [81.303; & & {$[1.043 ;$} & 2.539 \\
\hline & & 3.296] & & & 98.318] & 103.980] & $105.840]$ & 103.641] & 121.570] & $109.043]$ & 104.444] & & 19.868] & $0.361]$ \\
\hline 2 & & 0.759 & $14.647[2.492 ;$ & 14.307 [1.903; & 66.063 & 85.762 & 91.408 & 89.719 & 89.735 & 105.656 & 93.504 & & $2.690[-5.538 ;$ & $0.026[-$ \\
\hline & & $\begin{array}{l}{[0.091 ;} \\
2.741]\end{array}$ & 43.208] & 42.490] & $\begin{array}{l}{[49.206 ;} \\
82.701]\end{array}$ & $\begin{array}{l}{[74.980 ;} \\
97.432]\end{array}$ & $\begin{array}{l}79.782 ; \\
103.4991\end{array}$ & $\begin{array}{l}\text { [79.128; } \\
\text { 100.532] }\end{array}$ & $\begin{array}{l}\text { [74.434; } \\
\text { 105.2311 }\end{array}$ & $\begin{array}{l}\text { [94.954; } \\
116.999]\end{array}$ & $\begin{array}{l}{[82.628 ;} \\
\text { 104.87] }\end{array}$ & & 10.896] & $\begin{array}{l}\text { 1.599; } \\
1.661\end{array}$ \\
\hline différence & & 0.089 & -0.134 & 2.777 & $\begin{array}{l}02.1011 \\
12.477\end{array}$ & $\begin{array}{c}37.4526] \\
6.226\end{array}$ & $\begin{array}{c}10.407 \\
1.307\end{array}$ & $\frac{10.532]}{2.720}$ & $\frac{10.231]}{2.720}$ & $\begin{array}{c}11.60 .909] \\
-8.316\end{array}$ & $\begin{array}{c}104.827] \\
-0.809\end{array}$ & & 7.697 & $\begin{array}{l}.661] \\
-1.127\end{array}$ \\
\hline $\begin{array}{l}\text { estimee } \\
\text { Intervalle de }\end{array}$ & & & {$[-13.272$} & {$[-10.276 ; 20.859]$} & {$[-4,201]$} & {$[-6.459$} & {$[-12.982$.} & {$[-9.970$} & {$[-9,970$} & -21.145 & {$[-13.726$} & & {$[2.977$} & \\
\hline $\begin{array}{l}\text { Intervale de } \\
\text { confiance }\end{array}$ & & ; 1.244] & ;14.981] & $[-10.270,20.053]]$ & ;28.910] & ; 18.5900$]$ & 15.445] & ;15.171] & ; 15.171] & ; 4.060$]$ & ;11.728] & & ; 12.357$]$ & ; 0.635$]$ \\
\hline $\begin{array}{l}\text { Probabilitié de } \\
\text { différence }\end{array}$ & & $56.1 \%$ & $44.2 \%$ & $63.4 \%$ & $93.3 \%$ & $83.7 \%$ & $57.2 \%$ & $67.1 \%$ & $92.9 \%$ & $9.1 \%$ & $45.2 \%$ & & $99.8 \%$ & $10.0 \%$ \\
\hline
\end{tabular}

$\%=\%$ par rapport au côté controlatéral sain. $\mathrm{RUD}=$ radio-ulnaire distal $; \mathrm{F}=$ flexion. $\mathrm{E}=$ extension. $\mathrm{UI}=$ inclinaison ulnaire. $\mathrm{RI}=$ radiale. $\mathrm{P}=\mathrm{Pronation} . \mathrm{S}=$ Supination 